\title{
Highlights of top quark production measurements at the LHC
}

\author{
Shima Shimizu* on behalf of the ATLAS and CMS collaborations \\ High Energy Accelerator Research Organization (KEK), Japan \\ E-mail: shima.shimizu@cern.ch
}

\begin{abstract}
Cross-section measurements of the top quark production at the LHC are presented in this contribution. Several measurements of the top-quark pair production and the single top-quark production are performed at the ATLAS and CMS experiments using proton-proton collisions at a centreof-mass energy of $\sqrt{s}=13 \mathrm{TeV}$. The cross sections are measured differentially as a function of a variety of variables. The results are generally described by the Standard Model predictions, but the description of the next-to-leading-order Monte Carlo simulations is found to show deviations from the data.
\end{abstract}

XIV International Conference on Heavy Quarks and Leptons (HQL2018)

May 27- June 1, 2018

Yamagata Terrsa, Yamagata,Japan

${ }^{*}$ Speaker. 


\section{Introduction}

The top quark is the heaviest particle in the Standard Model and has a large coupling to the Higgs boson. It is expected to be involved in several new physics processes beyond the Standard Model and a precise measurement of the production cross section provides a test of the Standard Model.

At the Large Hadron Collider (LHC), there are two types of top-quark production, the topquark pair production and the single top-quark production. The former is a QCD process and $90 \%$ of the top-quark pair production at the LHC proceeds via $g g \rightarrow t \bar{t}$. The single top-quark production involves a weak process and contains a $W-t-b$ vertex. It proceeds via three channels, namely, $s$-channel, $t$-channel, and $t W$-channel with the associated production of a $W$ boson.

During the years 2015 and 2016, the ATLAS and CMS experiments [1, 2] collected protonproton collision data corresponding to an integrated luminosity of $36 \mathrm{fb}^{-1}$ at a centre-of-mass energy of $\sqrt{s}=13 \mathrm{TeV}$. The statistics of the data corresponds to the production of more than 30 million top-quark pairs. It allows several kinds of differential measurements and also measurements of processes with very small cross sections, providing an opportunity to examine various theoretical descriptions.

A top quark decays almost exclusively to a $b$ quark and a $W$ boson, which further decays to two quarks or a lepton and a neutrino. Therefore the signature of a top quark is a b-jet and either two jets or a lepton and missing transverse momentum. In the case of top-quark pair production, measurements are performed through dilepton channel, 1+jets channel, and all-hadronic channel, depending on the decay modes of $W$ bosons.

\section{Top-quark pair production}

Figure 1 shows a summary of the inclusive cross-section measurements of the top-quark pair production performed at the LHC and Tevatron. The measured data is well described by the theory lines. Several measurements are performed at $\sqrt{s}=13 \mathrm{TeV}$ using different channels, and they agree well with each other.

Figure 2 shows the fiducial cross section measured in the boosted regime, where a top quark is measured as a large size jet [4]. The data and the Monte Carlo (MC) predictions are compatible with each other, but the predictions are slightly higher than the data and have deviations from each other. Deviations are seen even for the predictions using the same next-to-leading-order (NLO) Matrix Element in the POWHEG generator. The differences come from the choice of the amount of gluon radiation and from different parton shower MCs.

Differential measurements provide valuable inputs for understanding and improvements of the MC description. Figure 3 shows the differential cross sections as a function of the $p_{\mathrm{T}}$ of the hadronically decaying top quark. The predictions tend to be harder than the data especially for the NLO MC predictions using PYTHIA for the parton shower simulation. This tendency is seen in both ATLAS and CMS measurements $[5,6]$ and for both particle-level and parton-level cross sections. The CMS experiment also measures the same cross sections in bins of the top-quark rapidity and sees no apparent rapidity dependence in the tendency of the harder MC descriptions. 


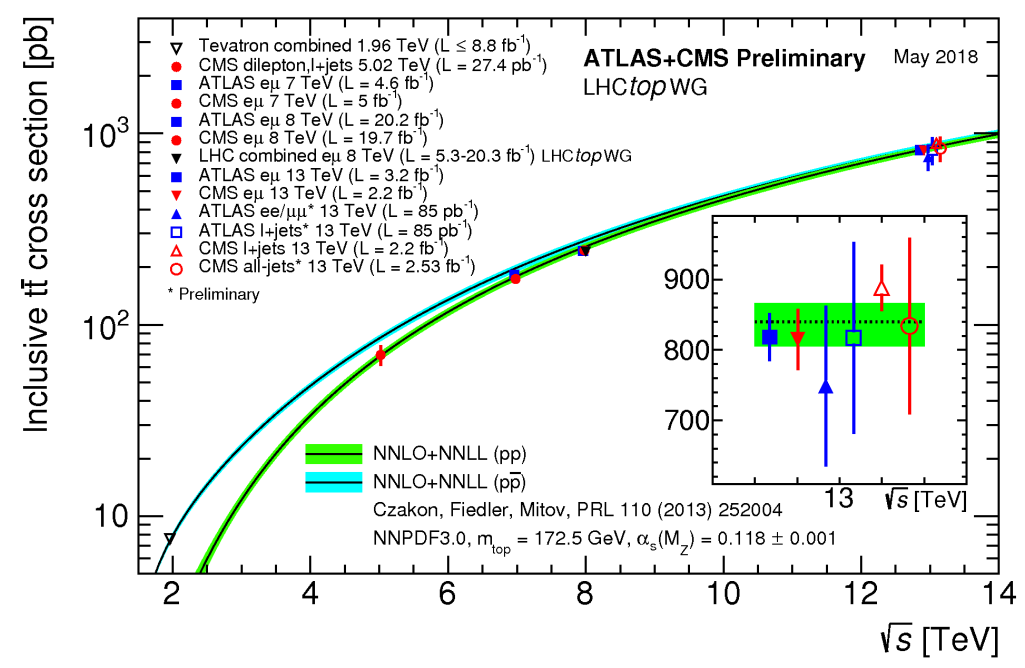

Figure 1: Summary of the LHC and Tevatron cross-section measurements of the top-quark pair production as a function of the centre-of-mass energy. The measurements are compared to the NNLO QCD calculation complemented with the NNLL resummation. [3].

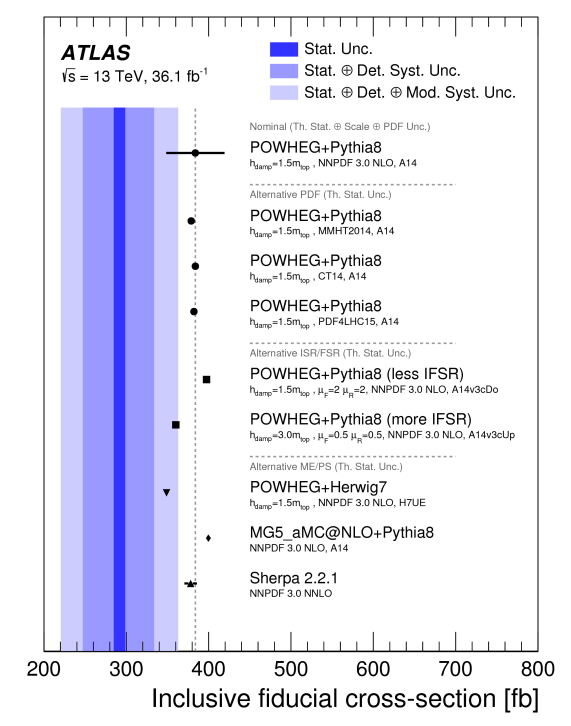

Figure 2: Fiducial cross section of the top-quark pair production with $p_{\mathrm{T}}>500$ $\mathrm{GeV}$ for the leading top quark and $p_{\mathrm{T}}>$ $350 \mathrm{GeV}$ for the subleading top quark. The measured data is compared to the MC predictions, where their inclusive cross sections are normalised to that of the NNLO+NNLL calculation. The systematic uncertainty in the theoretical prediction is shown only for the nominal POWHEG+Pythia8 prediction. Details are in [4].

The kinematics of the $t \bar{t}$ system is also interesting to be examined. For example, Figure 4 shows the normalised differential cross sections as a function of the mass of the $t \bar{t}$ system [7, 4]. The high mass region is well described by the MC predictions indicating no new physics up to the mass of $3 \mathrm{TeV}$. In the region with lower mass values, further improvement seems to be required in the $\mathrm{MC}$ prediction and in the QCD calculations.

Another approach is to measure the $t \bar{t}$ cross sections as a function of a number of kinematic variables without explicit reconstruction of the $t \bar{t}$ system as performed by the CMS experiment [8]. Events are considered if they contain the decay products of the lepton+jet channel, namely an isolated lepton and four jets including two b-jets. The cross section as a function of the sum of 

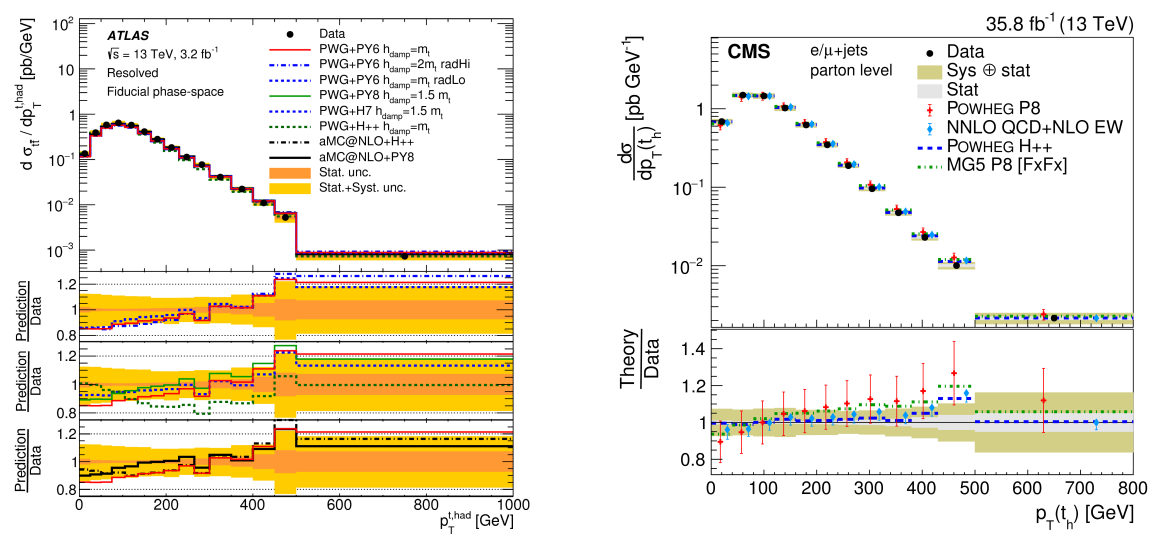

Figure 3: Differential cross sections of the top-quark pair production as a function of $p_{\mathrm{T}}$ of the hadronically decaying top quark measured in the lepton+jet channel. The cross sections after the hadronisation (particlelevel, left) is compared to the MC predictions and that at the top-quark level (parton-level, right) is compared to the MC predictions and the NNLO QCD + NLO EW calculation. [5] (left), [6] (right).
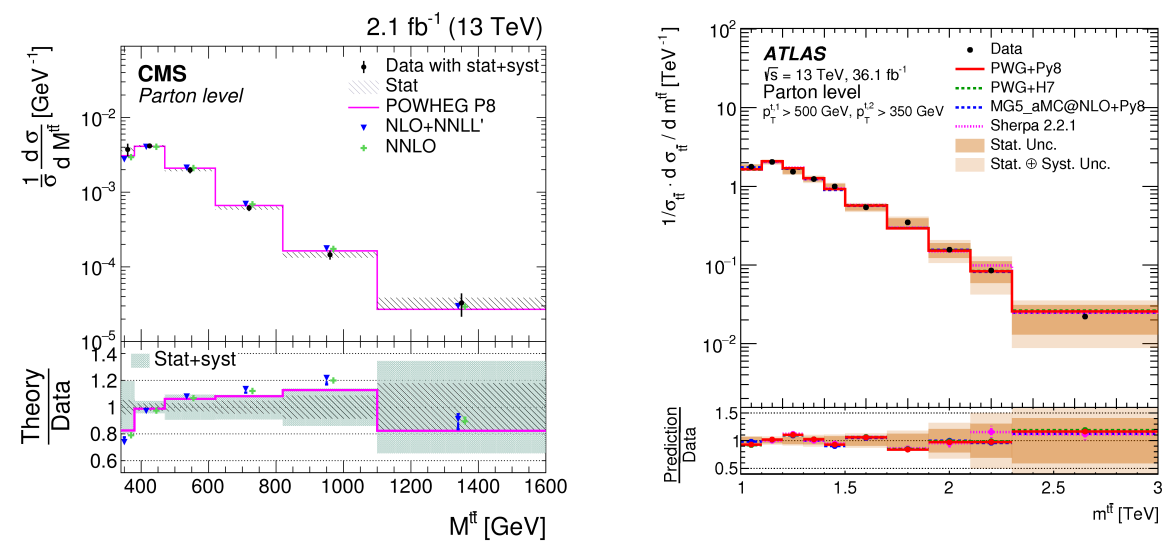

Figure 4: Normalised differential cross sections as a function of the mass of the $t \bar{t}$ system measured at parton-level. One is measured in the dilepton channel (left) and the other in the all-hadronic channel (right) using the amount of data indicated in the labels. [7] (left), [4] (right).

the jet $p_{\mathrm{T}}$ is shown in Figure 5. It shows that the nominal NLO MC prediction is compatible with data but the settings of the MC can result in different shapes. The results are expected to be useful inputs for MC tuning. As further inputs for MC tuning, the CMS experiment studied soft particle production in $t \bar{t}$ events [9] and jet substructure using the decay products of the top quarks [10]. Several MC settings are tested in these studies.

The LHCb experiment [11] also performed the measurement of the top-quark pair production in the forward region by requiring a muon, an electron, and a b-jet of the dilepton signature [12]. The cross section in the forward region is sensitive to the quark and gluon parton distribution functions in the large $x$ region, where $x$ is the momentum fraction of a quark or gluon in the proton. This region shows enhanced $q \bar{q} \rightarrow t \bar{t}$ production compared to the central region. The measured fiducial cross section is compatible with the Standard Model predictions but is slightly higher than the MC predictions with a difference of less than two sigmas. 


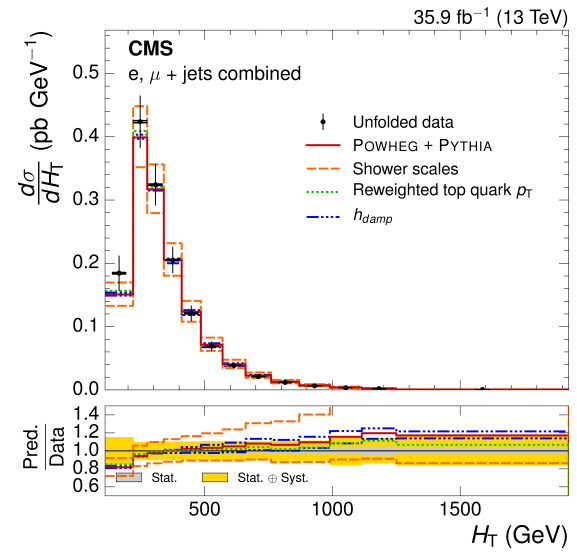

Figure 5: Differential cross section of top-quark pair production as a function of $H_{\mathrm{T}}$, the scalar sum of the jet $p_{\mathrm{T}}$ 's. Data is compared to the NLO MC prediction interfaced to the parton shower MC, POWHEG+PYTHIA, and the same predictions with reweighting of the top quark $p_{\mathrm{T}}$ or with different settings for the parton shower or the marching scale. [8].

\section{Single top-quark production}

Figure 6 shows a summary of the inclusive cross-section measurements of single top-quark production at the LHC. The results are in good agreement with the NLO+NNLL calculation. The $s$-channel production has a small cross section but is measured as non-zero with significances of $3.2 \sigma$ and $2.9 \sigma$ at $\sqrt{s}=8 \mathrm{TeV}$ by the ATLAS and CMS experiments, respectively. The CMS experiment has an updated result for the $t W$ production [13] at $\sqrt{s}=13 \mathrm{TeV}$. The measured cross section is $63.1 \pm 1.8$ (stat.) \pm 6.4 (syst.) \pm 2.1 (lumi.) pb, in agreement with the Standard Model predictions and with the previous results.

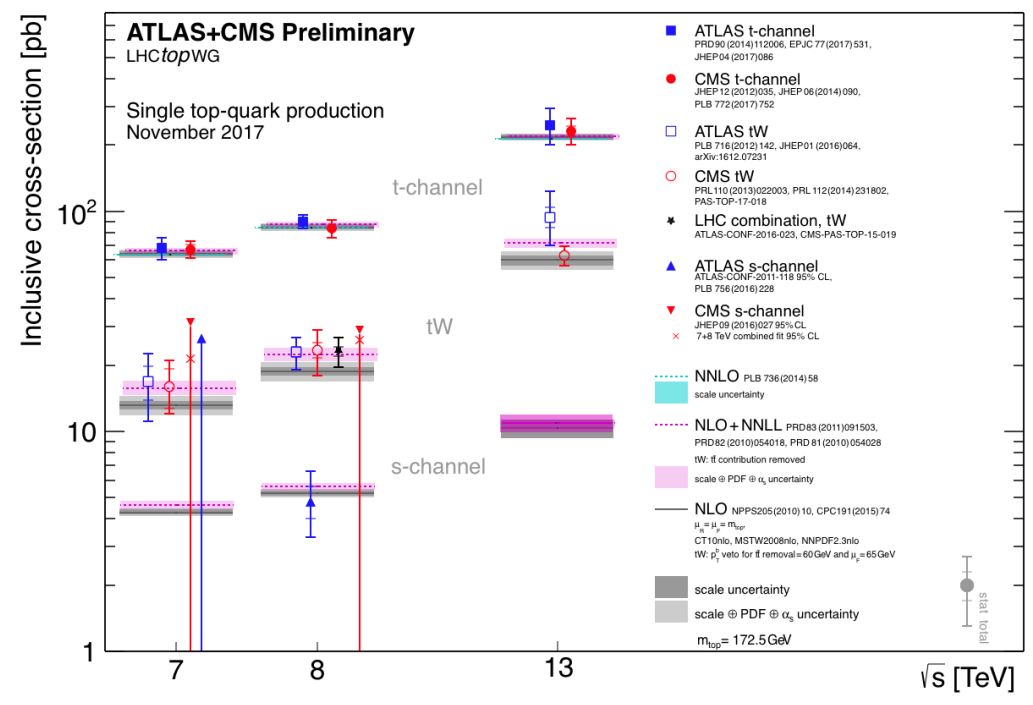

Figure 6: Summary of the ATLAS and CMS inclusive cross-section measurements of single top-quark production as a function of the centre-of-mass energy. They are shown separately for the different channels. The measurements are compared to the NLO QCD calculation and that complemented with the NNLL resummation. For the $t$-channel measurement, the NNLO QCD calculation is also compared. [3].

The ATLAS experiment performed a differential measurement of single top-quark production in the $t W$-channel [14]. The results are in good agreement with the MC predictions as in the example shown in Figure 7. However, there is a slope in the comparison of the central values 
which indicates that the MC predictions may underestimate the production of $t W$ decaying into high-momentum objects.

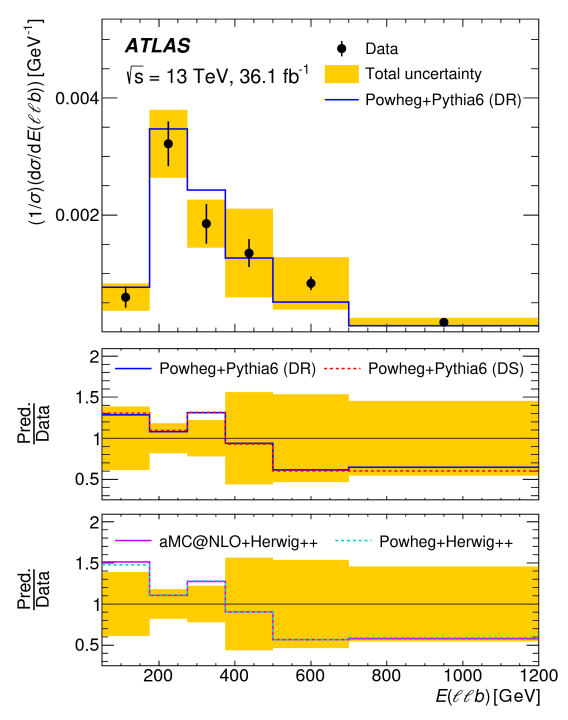

Figure 7: Normalised differential cross section of single top-quark production in association with a $W$ boson as a function of the energy of the system of two leptons and a b-jet. The data is compared to several NLO MC predictions. [14].

Single top-quark production in association with a $Z$ boson has a small cross section but is interesting as it involves processes with the $W W Z$ coupling and the $t Z$ coupling. The ATLAS and CMS experiments measured this production channel using leptonically decaying $W$ and $Z$ bosons $[15,16]$ in different fiducial phase spaces. Both results are consistent with the NLO predictions. The ATLAS result is $\sigma(t Z q)=600 \pm 170$ (stat.) \pm 140 (syst.) fb while the NLO prediction is $800_{-59}^{+49} \mathrm{fb}$, and the CMS result is $\sigma\left(t l^{+} l^{-} q, m_{l l}>30 \mathrm{GeV}\right)=123_{-31}^{+33}$ (stat. $)_{-23}^{+29}$ (syst.) fb while the NLO prediction is $94.2 \pm 3.1 \mathrm{fb}$.

\section{Summary}

The ATLAS, CMS and LHCb experiments performed several measurements of the top-quark production cross section having advantage of the large statistics available at the LHC. The measurements include various differential distributions and rare production channels. The results are generally well described by the Standard Model predictions. However, the NLO MC predictions show deviations from the data. The results of the production cross-section measurements are expected to provide inputs for further improvement of the MC description.

\section{References}

[1] ATLAS collaboration, The ATLAS Experiment at the CERN Large Hadron Collider, JINST 3 (2008) S08003.

[2] CMS collaboration, The CMS experiment at the CERN LHC, JINST 3 (2008) S08004.

[3] LHCtopWG Summary Plots, https:/twiki.cern.ch/twiki/bin/view/LHCPhysics/LHCTopWGSummaryPlots. 
[4] ATLAS collaboration, Measurements of $t \bar{t}$ differential cross-sections of highly boosted top quarks decaying to all-hadronic final states in pp collisions at $\sqrt{s}=13 \mathrm{TeV}$ using the ATLAS detector, submitted to PRD, [arXiv:1801.02052].

[5] ATLAS collaboration, Measurements of top-quark pair differential cross-sections in the lepton+jets channel in pp collisions at $\sqrt{s}=13$ TeV using the ATLAS detector, JHEP 11 (2017) 191 [arXiv:1708.00727].

[6] CMS collaboration, Measurement of differential cross sections for the production of top quark pairs and of additional jets in lepton+jets events from pp collisions at $\sqrt{s}=13$ TeV, Phys. Rev. D97 (2018) 112003 [arXiv:1803.08856].

[7] CMS collaboration, Measurement of normalized differential $\mathrm{t} \overline{\mathrm{t}}$ cross sections in the dilepton channel from pp collisions at $\sqrt{s}=13 \mathrm{TeV}$, JHEP 04 (2018) 060 [arXiv:1708. 07638].

[8] CMS collaboration, Measurements of differential cross sections of top quark pair production as a function of kinematic event variables in proton-proton collisions at $\sqrt{s}=13 \mathrm{TeV}$, JHEP 06 (2018) 002 [arXiv:1803.03991].

[9] CMS collaboration, Study of the underlying event in top quark pair production in pp collisions at 13 $\mathrm{TeV}$, submitted to EPJC, [arXiv:1807.02810].

[10] CMS collaboration, Measurement of jet substructure observables in $\mathrm{t} \overline{\mathrm{t}}$ events from pp collisions at $\sqrt{s}=13 \mathrm{TeV}$, CMS-PAS-TOP-17-013, https://cds.cern.ch/record/2308655, 2018.

[11] LHCb collaboration, The LHCb Detector at the LHC, JINST 3 (2008) S08005.

[12] LHCb collaboration, Measurement of forward top pair production in the dilepton channel in $p p$ collisions at $\sqrt{s}=13 \mathrm{TeV}$, submitted to JHEP, [arXiv:1803.05188].

[13] CMS collaboration, Measurement of the production cross section for single top quarks in association with $W$ bosons in proton-proton collisions at $\sqrt{s}=13 \mathrm{TeV}$, submitted to JHEP,

[arXiv:1805.07399].

[14] ATLAS collaboration, Measurement of differential cross-sections of a single top quark produced in association with a W boson at $\sqrt{s}=13$ TeV with ATLAS, Eur. Phys. J. C78 (2018) 186 [arXiv:1712.01602].

[15] ATLAS collaboration, Measurement of the production cross-section of a single top quark in association with a $\mathrm{Z}$ boson in proton-proton collisions at $13 \mathrm{TeV}$ with the ATLAS detector, Phys. Lett. B780 (2018) 557 [arXiv:1710.03659].

[16] CMS collaboration, Measurement of the associated production of a single top quark and a $Z$ boson in pp collisions at $\sqrt{s}=T e V$, Phys. Lett. B779 (2018) 358 [arXiv:1712.02825]. 